International Journal of Pure and Applied Mathematics

Volume 103 No. 2 2015, 347-358

ISSN: $1311-8080$ (printed version); ISSN: 1314-3395 (on-line version)

url: http://www.ijpam.eu

doi: http://dx.doi.org/10.12732/ijpam.v103i2.18

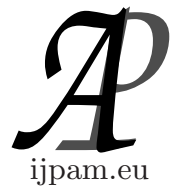

\title{
ANOTE ON THE GEODESIC-RAY PROPERTY IN MANIFOLDS WITHOUT CONJUGATE POINTS
}

\author{
Wedad Saleh ${ }^{1 \S}$, Adem Kiliçman ${ }^{2}$ \\ ${ }^{1}$ Department of Mathematics \\ University Putra Malaysia \\ 43400 UPM, Serdang, Selangor, MALAYSIA \\ ${ }^{2}$ Department of Mathematics and Institute for Mathematical Research \\ University Putra Malaysia \\ 43400 UPM, Serdang, Selangor, MALAYSIA
}

\begin{abstract}
Let $W^{n}$ be a $C^{\infty}$ complete, simply connected $n$-dimensional Riemannian manifold without conjugate points. The relationship between geodesicray property, starshapedness and convexity are established in $W^{n}$.Furthermore, some examples are given.
\end{abstract}

AMS Subject Classification: 52A20, 53C20

Key Words: convex set, manifolds without conjugate points, starshaped sets

\section{Introduction}

The behavior of geodesics in Riemannian manifolds without conjugate points as well as focal points has been studied by many geometers auch as [1], [3], [4], [6], [11], [13] and[14]. Hopf in a celebrated paper [12] proved that 2-dimensional tori without conjugate points are flat. So their universal coverings are flat planes where geodesics are straight lines. In 1994, Burago and Ivanov [5] generalized this result for $n$-dimensional tori. Green[10] in the late $1950_{s}$, proved the divergence of Jacobi fields and geodesic rays in the universal covering of compact surfaces without conjugate points. Green also proved the same result for com-

Received: June 11, 2015

(C) 2015 Academic Publications, Ltd.

$\S$ Correspondence author url: www.acadpubl.eu 
pact manifolds without conjugate points of any dimension. Eberlein [7]maked a complete proof of the divergence in manifolds $N$ regardless of dimension of $N$. On the other hand observes that the divergence might not be uniform, it could depend on the geodesic. Recently, Ruggiero[17] proved that geodesic rays in universal covering which meet an axis of a covering isometry diverge from this axis.

Motivated by earlier research work works $[15,16]$ and by the importance of the concept of geodesic rays, we establish the relationship between geodesic rays, starshapedness and convexity in Riemannian manifolds.

\section{Preliminaries}

In this section, we introduce some defintions and known results of Riemannian manifolds, which help us throughout the article. We refer to [21] for the standard material on differential geometry.

Let $N$ be a $C^{\infty} n$-dimensional Riemannian manifold, and $T_{z} N$ be the tangent space to $N$ at $z$. Also, assume that $\mu_{z}\left(x_{1}, x_{2}\right)$ is a positive inner product on the tangent space $T_{z} N\left(x_{1}, x_{2} \in T_{z} N\right)$, which is given for each point of $N$. Then, a $C^{\infty}$ map $\mu: z \longrightarrow \mu_{z}$, which assigns a positive inner product $\mu_{z}$ to $T_{z} N$ for each point $z$ of $N$ is called a Riemannian metric.

The length of a piecewise $C^{1}$ curve $\eta:\left[a_{1}, a_{2}\right] \longrightarrow N$ which is defined as follows:

$$
L(\eta)=\int_{a_{1}}^{a_{2}}\|\dot{\eta}(x)\| d x .
$$

We define $d\left(z_{1}, z_{2}\right)=\inf \left\{L(\eta): \eta\right.$ is a piecewise $C^{1}$ curve joining $z_{1}$ to $\left.z_{2}\right\}$ for any points $z_{1}, z_{2} \in N \cdot \nabla_{X} Y, X, Y \in N$ is a unique determined Riemannian connection which called Levi-Civita connection on every Riemannian manifolds. Furthermore, a smooth path $\eta$ is a geodesic if and only if its tangent vector is a prallel vector field along the path $\eta$, i.e, $\eta$ satisfies the equation $\nabla \dot{\eta}(t) \eta(t)=0$. Every path $\eta$ is joining $z_{1}, z_{2} \in N$ where $L(\eta)=d\left(z_{1}, z_{2}\right)$ is a minimal geodesic. Finally, assume that $(N, \eta)$ is a complete $n$-dimensional Riemannian manifold with Riemannian connection $\nabla$. Let $x_{1}, x_{2} \in N$ and $\eta:[0,1] \longrightarrow N$ be a geodesic joining the points $x_{1}$ and $x_{2}$, which means that $\eta_{x_{1}, x_{2}}(0)=x_{2}$ and $\eta_{x_{1}, x_{2}}(1)=x_{1}$.

Definition 1 (see[19]). A subset $B$ in a Riemannian manifold $N$ is convex if for each pair points $p, q \in N$, there is a unique minimal geodesic segment from $p$ to $q$ and this segment is in $B$. 
When dealing with a subset $B \subset W^{n}$, where $W^{n}$ is a $C^{\infty}$ complete, simply connected $n$ - dimensional Riemannian manifold without conjugate points, the word " a unique minimal geodesic segment" should be replaced by " the geodesic segment".

Definition 2 (see[18]). A subset $S$ in a Riemannian manifold $N$ is starshaped if there is a point $p \in S$ such that for all $q \in S$ there is a unique minimal geodesic segment $\gamma_{p q}$ from $p$ to $q$ and this segment is in $S$. In such a case, the set $S$ is starshaped with respect to $p$ or $p$ sees $S$ via $S$.

Remark 1. The subset of $S$ consisting of all points like $p$ is called the kernel of $S(\operatorname{ker} S)$. In $W^{n}$, a subset $S$ is starshaped if there is a point $p \in S$ such that for all $q \in S$, the geodesic segment $\gamma_{p q}$ joining $p$ and $q$ is contained in $S$.

Let $W_{1}$ and $W_{2}$ be complete, simply connected $C^{\infty}$ Riemannian manifolds without conjugate points. Assume that $W_{1} \times W_{2}$ is a complete, simply connected $C^{\infty}$ Riemannian manifold without conjugate points. Notice that $\operatorname{dim}\left(W_{1} \times\right.$ $\left.W_{2}\right)=\operatorname{dim}\left(W_{1}\right)+\operatorname{dim}\left(W_{2}\right)$. Consequently, each pair of different points $p=$ $\left(p_{1}, p_{2}\right)$ and $q=\left(q_{1}, q_{2}\right)$ in $W_{1} \times W_{2}$ are joined by a unique geodesic segment $\gamma$. This segment when naturally projected on $W_{1}$ and $W_{2}$ yields two geodesic segments joining $p_{i}$ and $q_{i}, i=1,2$ each one is unique in its own manifold. The natural projection will be denoted by $\varphi_{i}: W_{1} \times W_{2} \longrightarrow W_{i}$ where $\varphi_{i}\left(p_{1}, p_{2}\right)=p_{i}, i=1,2($ see $[2])$.

In 1974, Stavrakas [20] defined the half-ray property in Euclidean space as follows:

Definition 3. let $S \subset \mathbb{R}^{n}$, and let $S^{c}$ be the complement of $S . S$ has the half-ray property if and if for every $x \in S^{c}$ there exists a half line $L$ with $x$ as vertex $x$ such that $L \cap S=\phi$.

In the same paper there are some geometric properties of starshaped in Euclidean space were considered as follows: let $S \subset \mathbb{R}^{n}, n \geq 2$, be compact and suppose that $\cap_{x \in E(x)} S(x) \neq \phi$. Then, the following are equivalent:

1. $S$ has the half- ray property.

2. $\operatorname{ker} S=\cap_{x \in E(x)} S(x)$.

Also, Goodey in [8] used the half-ray property to prove the following theorem:

Theorem 4. If $S \subset \mathbb{R}^{n}$ is a nonseparating ( its complement is connected) compact set and $\cap\{S(y): y \in E(S)\} \neq \phi$, where $E(S)$ is the totality of $(n-2)$ extreme points of $S$, then $S$ is starshaped set. 


\section{The Geodesic-Ray Property}

Let us start by defining the geodesic-ray property in a $C^{\infty}$ complete Riemannian manifold $N$. Let $\gamma:(-\infty, \infty) \longrightarrow N$ be a maximal geodesic. Let $m \in N$ be a point at $\gamma$ such that $\gamma(0)=m$ and $\gamma(0)=X \in T_{m} N$. The geodesic $\gamma$ can be divided at two half geodesic rays $\gamma=\gamma_{+} \cup \gamma_{-}$where $\gamma_{+}:[0, \infty)$ such that $\gamma_{+}^{\prime}(0)=X$ and $\gamma_{-}:(-\infty, 0]$ such that $\gamma_{-}^{\prime}(0)=-X$

Definition 5. Let $B \subset N$ and let $B^{c}$ be the complement of $B$. We say that $B$ has the geodesic-ray property if for all $x \in B^{c}$ and for any geodesic $\gamma=\gamma_{+} \cup \gamma_{-}$such that $\gamma(0)=x$, then one or both of two half geodesic rays $\gamma_{+}$ or $\gamma_{-}$has empty intersection of $B$.

In the light of definition (5), we can prove that the geodesic-ray property is an intersection property in the following proposition:

Proposition 1. The intersection of two sets have the geodesic-ray property has the geodesic-ray property

Proof. Let $B_{1}, B_{2} \subset N$ have the geodesic-ray property. Let us assume, on the contrary, that $B_{1} \cap B_{2}$ has no the geodesic-ray property. Hence, there exists a point $x \in\left(B_{1} \cap B_{2}\right)^{c}=B_{1}^{c} \cup B_{2}^{c}$ and a geodesic $\gamma=\gamma_{+} \cup \gamma_{-}$, where $\gamma(0)=x$, such that the two rays $\gamma_{+}$and $\gamma_{-}$have no empty intersection with $B_{1} \cap B_{2}$. Since $x \in B_{1}^{c}$ or $x \in B_{2}^{c}$, then either $B_{1}$ or $B_{2}$ has no geodesic-ray property which is a contradiction.

Remark 2. In general, the union of geodesic-ray property is not geodesicray property.

Example 1. Let $A$ be the union of two closed balls $D_{1}=B(a, r)$ and $D_{2}=B(b, r)$ in $\mathbb{R}^{n}$ of equal radii $r$ and center $a, b$ in $\mathbb{R}^{n}$ such that $D_{1} \cap D_{2}=\phi$. It is clear that $A$ is the union of a two line property. Let $c=\frac{(a+b)}{2} \in \mathbb{R}^{n}$, then the half line $\overrightarrow{(c a)}$ starting from $c \in \mathbb{R}^{n}$ and passing throw $a \in \mathbb{R}^{n}$ has no empty intersection with $D_{1} \subset A$. Also, the half line $\overrightarrow{(c b)}$ has no empty intersection with $D_{2} \subset A$. Since $c \in A^{c}$, then $A$ has no line property.

Theorem 6. If $p$ is an interior point in a closed subset $B \subset W^{n}$ with smooth boundary $\partial B$ and $B$ has the geodesic-ray property, then each geodesic ray from $p$ intersects the hypersurface $\partial B$ exactly at one point and the intersection is transversal.

Proof. Let us consider an arbitrary point $q \in \partial B$. Firstly, we show that the geodesic ray $\eta$ through $p$ and $q$ should intersect $\partial B$ at $q$ transversally. As- 
sume that the intersection is tangential as indicated in Figure 1. Draw a thin geodesic cone $C$ with vertex at $q$ and axis $\eta$ whose base is included in $U(p) \subset B$, where $U(p)$ is an open neighborhood about $p$. From the figure it becomes clear that there exists a geodesic $\gamma=\gamma_{+} \cup \gamma_{-}$through a point $x \in W^{n} \backslash B$ such that $\gamma_{x q} \subset \gamma_{+}$and $\gamma_{p} x \subset \gamma_{-}$where $\dot{p} \in U(p)$. Consequently, $\gamma_{+} \cap B \neq \phi$ and $\gamma_{-} \cap B \neq \phi$ which contradicts $B$ has the geodesic-ray property.

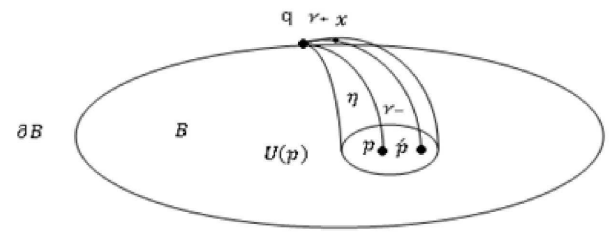

Figure 1: The geodesic-ray $\eta$ intersects $\partial B$ at $q$ tangentially

To complete the proof assume that there exists a geodesic ray $\eta$ from $p$ which intersects $\partial B$ twice, then we shall arrive at Figure 2. Hence, there exists a geodesic $\gamma=\gamma_{+} \cup \gamma_{-}$through a point $x \in W^{n} \backslash B$ such that $\gamma_{q x} \subset \gamma_{+}$where $q \in \partial B$ and $\gamma_{x s} \subset \gamma_{-}$where $s \in \partial B$. Consequently, $\gamma_{+} \cap B \neq \phi$ and $\gamma_{-} \cap B \neq \phi$ which contradicts $B$ has the geodesic-ray property.

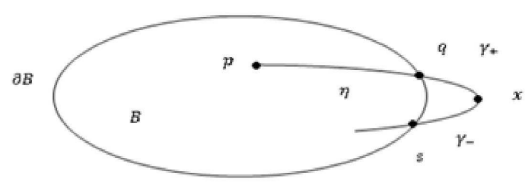

Figure 2: The geodesic $\eta$ intersects $\partial B$ twice

Corollary 7. Let $B$ be a closed subset in $W^{n}$ with smooth boundary $\partial B$. If $B$ has the geodesic-ray property, then each tangent geodesic $\eta$ to $\partial B$ has the property $\eta \cap \operatorname{Int}(B)=\phi$.

Corollary 8. Let $B$ be a closed subset in $W^{n}$ with smooth boundary $\partial B$ and $B$ has the geodesic-ray property. If $p \in \partial B$, then $B$ lies on one side of the tangent geodesic hypersurface of $\partial B$ at $p$. 


\section{Starshapedness and Geodesic - ray Property}

In this section, we study the relationship between starshapedness in a $C^{\infty}$ complete, simply connected Riemannian manifold without conjugate points and geodesic-ray property.

Theorem 9. Let $S \subset W^{n}$ starshaped set . $S$ has the geodesic- ray property if and if $S=k e r S$.

Proof. Let $S=\operatorname{ker} S$. Now, we want to prove that $S$ has the geodesic-ray property. Suppose that $S$ does not have the geodesic- ray property. So, there is $x \in S^{c}$ and $x=\gamma_{+}(0)=\gamma_{-}(0)$ such that $\gamma_{+} \cap S \neq \phi$ and $\gamma_{-} \cap S \neq \phi$. Then, there exists two different points $p, q \in S$ such that $p \in \gamma_{+} \cap S$ and $q \in \gamma_{-} \cap S$. Consequently, $\gamma_{p x} \subset \gamma_{+}$and $\gamma_{x q} \subset \gamma_{-}$which means that the geodesic segment $\gamma_{p q}$ joining $p$ and $q$ is not included in $S$ and hence $p$ does not see $q$ via $S$. This is a contradiction to the assumption that $S=\operatorname{ker} S$. Assume on the contrary that $S \neq \operatorname{ker} S$, then there is two points $p, q \in S$ such that $p$ does not see $q$ via $S$. Thus, the geodesic segment $\gamma_{p q}$ joining $p$ and $q$ is not contained in $S$. Then, there is a point $x \in \gamma_{p q}$ such that $x \notin S$. This implies that there exists a geodesic $\gamma=\gamma_{+} \cup \gamma_{-}$through a point $x \in S^{c}$ and $x=\gamma_{+}(0)=\gamma_{-}(0)$ such that $\gamma_{p x} \subset \gamma_{+}$and $\gamma_{x q} \subset \gamma_{-}$. Then, $\gamma_{+} \cap S \neq \phi$ and $\gamma_{-} \cap S \neq \phi$. This argument shows that $S$ does not have the geodesic-ray property.

Theorem 10. A closed starshaped set $S \in W^{n}$ has the geodesic-ray property if and only if $\partial S \subset k e r S$.

Proof. If $S$ has the geodesic-ray property, then $\operatorname{ker} S=S \supset \partial S$. Now, let $\partial S \subset \operatorname{ker} S$, we want to prove that $S$ has the geodesic-ray property. Suppose that $S$ is not , then there is $x \in S^{c}$ and $x=\gamma_{+}(0)=\gamma_{-}(0)$ such that $\gamma_{+} \cap \partial S \neq \phi$ and $\gamma_{-} \cap \partial S \neq \phi$.This implies that there is $p \in \gamma_{+} \cap \partial S$ and $q \in \gamma_{-} \cap \partial S$ such that $\gamma_{p q} \subset \gamma$ and $\gamma_{p q} \cap S=\phi$ and hence $p$ does not see $q$ via $S$ which contradicts the fact that $\partial S \subset \operatorname{ker} S$ and so $S$ has geodesic-ray property.

Theorem 11. Assume that $S$ is an open connected subset of $W^{2}$. Then, ker $S$ is the set of all points of maximal visibility.

Proof. Let us consider that $Z$ is the set of all points of maximal visibility in $S$. We want to prove that $Z=\operatorname{ker} S$. It is clear that $\operatorname{ker} S \subset Z$, so we will show that $Z \subset \operatorname{ker} S$. Let $x \notin \operatorname{ker} S$ and $x \in Z$. Then, there exists a geodesic $\gamma=\gamma_{+} \cup \gamma_{-}$through a point $x \in Z$ and $x=\gamma_{+}(0)=\gamma_{-}(0)$ such that $\gamma_{+} \cap \operatorname{ker} S \neq \phi$ and $\gamma_{-} \cap \operatorname{ker} S \neq \phi$.Then, there exists two different points 
$p, q \in \operatorname{ker} S$ such that $p \in \gamma_{+} \cap \operatorname{ker} S$ and $q \in \gamma_{-} \cap \operatorname{ker} S$. Consequently, $\gamma_{p x} \subset \gamma_{+}$and $\gamma_{x q} \subset \gamma_{-}$. This implies that the geodesic segment $\gamma_{p q}$ joining $p$ and $q$ is not included in ker $S$. This argument shows that $k e r S$ is non-convex which contradiction. Then, $\operatorname{ker} S \subset Z$ and the proof is complete.

\section{Convexity and the Geodesic-Ray Property}

In this section, we aim to give the relationship between convex and the geodesic-ray property in a Riemannian manifold.

Theorem 12. Let $B$ be an open subset in a $C^{\infty}$ complete $n$-dimensional Riemannian manifold $N$. If $B$ has the geodesic-ray property, , then $B$ is convex.

Proof. Assume on the contrary that $B$ is not convex (see Figure 3).Then, there exists a pair of points $p, q \in B$ such that the geodesic segment $\gamma_{p q}$ joining $p$ and $q$ is not contained in $B$. Thus, there is a point $x \in \gamma_{p q}$ such that $x \notin B$. So, there exists a geodesic $\gamma=\gamma_{+} \cup \gamma_{-}$through a point $x \in B^{c}$ and $x=\gamma_{+}(0)=\gamma_{-}(0)$ such that $\gamma_{p x} \subset \gamma_{+}$and $\gamma_{x q} \subset \gamma_{-}$. Then, $\gamma_{+} \cap B \neq \phi$ and $\gamma_{-} \cap B \neq \phi$. This argument shows that $B$ does not have the geodesic-ray property contradicting the hypothesis.

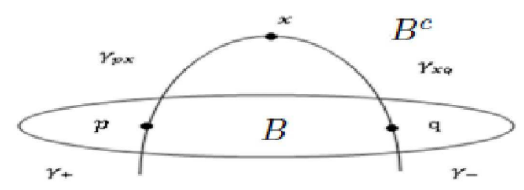

Figure 3: $B$ is not convex

The converse of the last Theorem 12 is not generally true even in genaral Riemannian manifold. The following example indicates this claim.

Example 2. Let $B(p, r), 0<r<\frac{\pi}{2}$ be an open geodesic ball in the unit sphere $S^{n} \subset \mathbb{R}^{n+1}$ centered at the north pole $p$ with radius $0<r<\frac{\pi}{2}$ (see Figure 4). Let $q_{1}$ and $q_{2}$ be two arbitrary points in $B$, then there exists a geodesic $\gamma=\gamma_{+} \cup \gamma_{-}$through a point $x \in B^{c}$ and $x=\gamma_{+}(0)=\gamma_{-}(0)$ such that $\gamma_{q_{1} x} \subset \gamma_{+}$and $\gamma_{x q_{2}} \subset \gamma_{-}$. Thus, $\gamma_{+} \cap B \neq \phi$ and $\gamma_{-} \cap B \neq \phi$. This implies that $B$ does not have the geodesic-ray property while $B(p, r)$ is convex subset. 


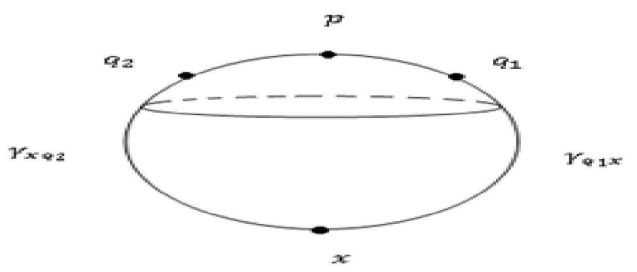

Figure 4: $B(p, r), 0<r<\frac{\pi}{2}$

Theorem 13. Let $B \subset W^{n}$ be an open subset. $B$ has the geodesic-ray property if and only if $B$ is convex.

Proof. Let $B \subset W^{n}$ be a convex subset which does not have the geodesicray property. Then, there exists a geodesic $\gamma=\gamma_{+} \cup \gamma_{-}$through a point $x \in B^{c}$ and $x=\gamma_{+}(0)=\gamma_{-}(0)$ such that $\gamma_{+} \cap B \neq \phi$ and $\gamma_{-} \cap B \neq \phi$. There exists two different points $p, q \in B$ such that $p \in \gamma_{+} \cap B$ and $q \in \gamma_{-} \cap B$. Consequently, $\gamma_{p x} \subset \gamma_{+}$and $\gamma_{x q} \subset \gamma_{-}$such that the geodesic segment $\gamma_{p q}$ joining $p$ and $q$ is not included in $B$ (see Figure 5). This argument shows that $B$ is non-convex contradicting the hypothesis.

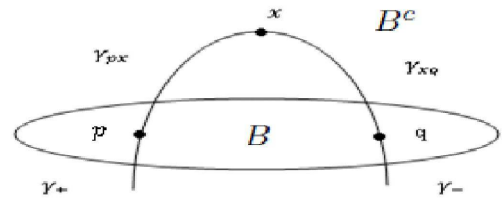

Figure 5: $B$ does not have the geodesic-ray property

The proof of another side can be carried out similar to that of Theorem 12 .

Remark 3. Let $B$ be a closed subset in a $C^{\infty}$ complete, simply connected $n$ - dimensional Riemannian manifold without conjugate points $W^{n}$.If $B$ is a strictly convex, then $B$ has the geodesic-ray property.

Now, we give the following example of set which has the geodesic-ray property but it is not strictly convex.

Example 3. Let $B \subset \mathbb{R}^{2}$ be a closed subset. Then, $B$ has the line property but it is not strictly convex (see Figure 6). 


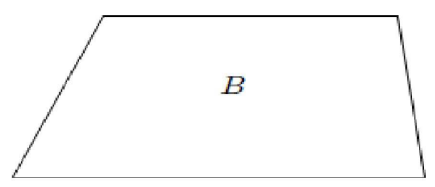

Figure 6: $B$ has the line property but it is not strictly convex

\section{Geodesic -ray Property in Riemannian Manifolds Product}

Geodesic-ray property in the Cartesian product of two complete, simply connected Riemannian manifolds without conjugate points is given by the following theorem:

Theorem 14. Let $B_{1} \subset W_{1}$ and $B_{2} \subset W_{2}$ where $W_{1}$ and $W_{2}$ are $C^{\infty}$ complete, simply connected Riemannian manifolds without conjugate points. $B_{1} \times B_{2}$ has geodesic-ray property if and only if $B_{1}$ and $B_{2}$ have the geodesicray property.

Proof. Firstly, assume that $B_{1} \times B_{2}$ has the geodesic-ray property of $W_{1} \times$ $W_{2}$. Suppose that $B_{1}$ and $B_{2}$ do not have the geodesic-ray property. Then, there exist a geodesic segment $\gamma^{1}=\gamma_{+}^{1} \cup \gamma_{-}^{1}$ through a point $x_{1} \in B_{1}^{c}$ and $x_{1}=$ $\gamma_{+}^{1}(0)=\gamma_{-}^{1}(0)$ such that $\gamma_{+}^{1} \cap B_{1} \neq \phi$ and $\gamma_{-}^{1} \cap B_{1} \neq \phi$. So, there exists two different points $p_{1}, q_{1} \in B_{1}$ such that $p_{1} \in \gamma_{+}^{1} \cap B_{1}$ and $q_{1} \in \gamma_{-}^{1} \cap B_{1}$. Consequently, $\gamma_{p_{1} x_{1}}^{1} \subset \gamma_{+}^{1}$ and $\gamma_{x_{1} q_{1}}^{1} \subset \gamma_{+}^{1}$, then the geodesic segment $\gamma_{p_{1} q_{1}}^{1} \not \subset B_{1}$. Since $B_{2}$ does not have the geodesic-ray property. Thus, there exists a geodesic $\gamma^{2}=\gamma_{+}^{2} \cup \gamma_{-}^{2}$ through a point $x_{2} \in B_{2}^{c}$ and $x_{2}=\gamma_{+}^{2}(0)=\gamma_{-}^{2}(0)$ such that $\gamma_{+}^{2} \cap B_{2} \neq \phi$ and $\gamma_{-}^{2} \cap B_{2} \neq \phi$. Then, there exist two different points $p_{2}, q_{2} \in B_{2}$ such that $p_{2} \in \gamma_{+}^{2} \cap B_{2}$ and $q_{2} \in \gamma_{-}^{2} \cap B_{2}$. Consequently, $\gamma_{p_{2} x_{2}}^{2} \subset \gamma_{+}^{2}$ and $\gamma_{x_{2} q_{2}}^{2} \subset \gamma_{+}^{2}$ ,then the geodesic segment $\gamma_{p_{2} q_{2}}^{2} \not \subset B_{2}$. Let $p=\left(p_{1}, p_{2}\right), q=\left(q_{1}, q_{2}\right) \in B_{1} \times B_{2}$. We can claim that $\gamma_{p q} \not \subset B_{1} \times B_{2}$. Let $x=\left(x_{1}, x_{2}\right)=\gamma_{+}(0)=\gamma_{-}(0)$, where $\gamma_{p x} \subset \gamma_{+}$and $\gamma_{x q} \subset \gamma_{-}$, but $\gamma_{+} \cap\left(B_{1} \times B_{2}\right) \neq \phi$ and $\gamma_{-} \cap\left(B_{1} \times B_{2}\right) \neq \phi$. This argument shows that $B_{1} \times B_{2}$ has the geodesic-ray property.

Now, let $B_{1} \times B_{2}$ does not have the geodesic-ray property, then there exist a geodesic $\gamma=\gamma_{+} \cup \gamma_{-}$through a point $x=\left(x_{1}, x_{2}\right) \in\left(B_{1} \times B_{2}\right)^{c}$ and $x=$ $\gamma_{+}(0)=\gamma_{-}(0)$ such that $\gamma_{+} \cap\left(B_{1} \times B_{2}\right) \neq \phi$ and $\gamma_{-} \cap\left(B_{1} \times B_{2}\right) \neq \phi$. Then, there exist two different points $p=\left(p_{1}, p_{2}\right), q=\left(q_{1}, q_{2}\right) \in B_{1} \times B_{2}$ such that $p \in \gamma_{+} \cap\left(B_{1} \times B_{2}\right)$ and $q \in \gamma_{-} \cap\left(B_{1} \times B_{2}\right)$. Consequently, $\gamma_{p x} \subset \gamma_{+}$and $\gamma_{x q} \subset \gamma_{-}$. 
Then, the geodesic segment $\gamma_{p q} \not \subset B_{1} \times B_{2}$. Since $\left(x_{1}, x_{2}\right) \notin\left(B_{1} \times B_{2}\right)$, we have that one - at least- of the following statements $x_{1} \notin B_{1}, x_{2} \notin B_{2}$. Thus, $x_{1} \in B_{1}^{c}$ , $\gamma_{p_{1} x_{1}} \subset \gamma_{+}^{1}$ and $\gamma_{x_{1} q_{1}} \subset \gamma_{-}^{1}$ this implies that $\gamma_{+}^{1} \cap B_{1} \neq \phi$ and $\gamma_{-}^{1} \cap B_{1} \neq \phi$. This argument shows that $B_{1}$ does not have the geodesic-ray property.

The following example may be considered as an application of Theorem 14 .

Example 4. Consider $B_{1} \subset \mathbb{R}^{1}$ and $B_{2} \subset \mathbb{R}^{2}$ where $B_{1}=[0,1]$ and $B_{2}=\left\{\left(x_{1}, x_{2}\right): x_{1}^{2}+x_{2}^{2} \leq 1\right\}$ is the unit disc. It is clear that $B_{1}$ and $B_{2}$ have the line property. Also, $B_{1} \times B_{2} \subset \mathbb{R}^{1} \times \mathbb{R}^{2}=\mathbb{R}^{3}$ which represents a truncated cylinder has the line property.

In the light of Theorem 14 we can prove the following corollary:

Corollary 15. Assume that $B \subset W_{1} \times W_{2}$ has the geodesic-ray property . Then, the natural projections $B_{i}=\varphi_{i} B \subset W_{i}$ of $B$ onto $W_{i}, i=1,2$ have the geodesic-ray property.

It is worth mentioning that the converse of the above corollary is not necessarily true, i.e., for a subset $B \subset W_{1} \times W_{2}$ the natural projections $\varphi_{i} B \subset$ $W_{i}, i=1,2$ might have the geodesic-ray property although $B$ does not have the geodesic-ray property. Figure 7 shows this fact. Notice that in this case, $\varphi_{1} B \times \varphi_{2} B \neq B$.

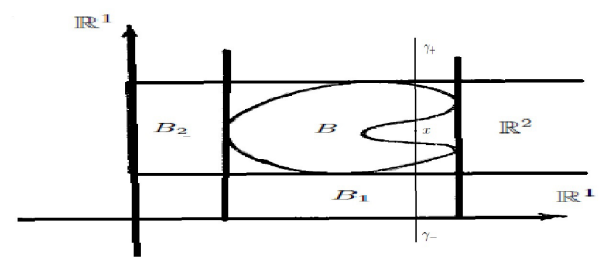

Figure 7: $B_{1}$ and $B_{2}$ have the line property but $B$ does not have

\section{Conclusion}

All results of the present work are valid in Euclidean space $\mathbb{R}^{n}$ as a manifolds without conjugate points [9]. On the other hand, the generalization of Theorem11 to $W^{n}, n \geq 3$ is more difficult and is left as open problem. 


\section{Acknowledgement}

The authors are exceptionally grateful to the anonymous referees for their valuable suggestions and comments, which helped the authors to improve the work.

\section{References}

[1] Adem Kilicman , Wedad Saleh , A Note on Starshaped Sets in2Dimensional Manifolds without Conjugate Points,Journal of Function Spaces, 2014(2014),1-3.

[2] M. Beltagy , Convex and Starshaped subsets in Manifolds Product, Communications, Faculty of Science, University of Ankara Series A1 Mathematics and Statistics, 41(1992),35-44 .

[3] M. Beltagy, Exposed points and convexity,Indian Journal of pure and applied mathematics, 24(1993),111-111 .

[4] M. Beltgy, S. Shenawy, A. Elsharkawy, Convexity and two piece property in $S^{n}$,International Journal of Pure and Applied Mathematics,95, No.3 (2014), 347-356.

[5] D.Burago,S.Ivanov, Riemannian tori without conjugate points are flat, $G e$ ometric and Functional Analysis, 4, No.3 (1994), 259-269.

[6] Patrick Eberlein, Geodesic flows in certain manifolds with no conjugate points, Transactions of the American Mathematical Society, 167(1972), 151-170.

[7] P.Eberlein, When is a geodesic flow of Anosov type I, J. Differ. Geom., 8(1973), 437-463.

[8] Paul R. Goodey, A Note on Starshaped Sets, Pacific journal of mathematics, 61, No.1 (1975), 151-152.

[9] S.M.Goto, Manifolds without conjugate points, J. Diff. Geom., 13(1978), 341-359.

[10] L.W.Green, Geodesic instability, Proceedings of the American Mathematical Society, 7(1956), 438-448 . 
[11] Gustav A. Hedlund , Geodesics on a two dimensional Riemannian manifolds with periodic coefficients, The Annals of Mathematics, 33, No.4(1932), 719-739.

[12] E.Hopf, Closed surfaces without conjugate points, Proceeding of the $\mathrm{Na}$ tional Academy of Sciences, 34(1948), 47-51.

[13] Akhlad Iqbal, Shahid Ali, I. Ahmad, On geodesic E-convex sets, geodesic E-convex functions and E-epigraphs, Journal Optimization Theory and Applications, 155, No.1(2012),239-251.

[14] H.B.Pandey, Cartesian product of two manifolds, Indian Journal of pure and applied mathematics, 12, No.1 (1981),55-60.

[15] R.Ruggiero, Expansive geodesic flows in manifolds without conjugate points, Ergodic Theory and Dynamical Systems, 17(1997), 211-225.

[16] R.Ruggiero, Weak stability properties of the geodesic flow and global hyperbolic geometry, a survey,Preprint PUC-Rio (2006).

[17] R.Ruggiero, A note on the divergence of geodesic rays in manifolds without conjugate points, Geometriae Dedicata., 134(2008), 131-138 .

[18] G. Santhanam , Isoperimetric upper bounds for the first eigen value,Proceeding Mathematical Sciences, 122 ,No.3(2012), 375-384 .

[19] Seydehsomayeh Hosseini, Mohamed R. Pouryaevali , On The Metric Projection onto Prox-regular Subsets of Riemannian Manifolds, Proceedings of the American Mathematical Society, 141, No. 1(2013), 233-244 .

[20] N.Stavrakas, A Note on Starshaped Sets, (k)-extreme Points and the Half Ray Property,Pacific Journal of Mathematics, 53(1974), 627-628.

[21] Constantin Udrist, Convex Funcions and Optimization Methods on Riemannian Manifolds, Kluwer Academic (1994). 